J. Jpn. Soc. Comp. Statist., 18(2005), 33-46

\title{
ESTIMATION FROM PSEUDO PARTIAL LIKELIHOOD IN A SEMIPARAMETRIC CURE MODEL
}

\author{
Tomoyuki Sugimoto*, Toshimitsu Hamasaki ${ }^{\dagger}$ and Masashi Goto ${ }^{\ddagger}$
}

\begin{abstract}
We consider a cure model identical to one discussed by Kuk and Chen (1992), Sy and Taylor (2000) and Peng and Dear (2000). The feature of this model is that one uses the logistic regression model for the cure rate and Cox's proportional hazards model for the latent distribution. We propose a new semiparametric estimation method in this model using a criterion named the pseudo partial likelihood. Simulation studies show that the proposed method is appropriate for practical use, compared with semiparametric estimation via the EM algorithm. An application to data from a breast cancer with three treatment arms of adjuvant therapy is given to illustrate the aspect of the proposed method.
\end{abstract}

\section{Introduction}

The Cox model (Cox, 1972) is the most typical tool for exploring the effects of covariates on the survival distribution. Although a proportionality assumption in linear modelling with the Cox process is easily violated in complex survival data, it is possible to provide a useful summary or prediction of such data by incorporating some flexibility into the relative score function for the Cox model. Still, there exist cases where further statistical interpretations are needed, apart from results obtained by some flexible modifications on the relative score function for the Cox model. One example from a medical viewpoint is the statistical summaries of a cure rate.

The Kaplan-Meier estimate sketched from a database of long follow-up of cancer patients may often have a long and flat tail if the event of death from cancer is of specific interest. Then long-term survivors who live healthily without any recurrence over a certain period may be chosen as cured individuals in phenomenal meaning or empirical evidence. From such a point of view, the statistical approaches to estimate a cure rate have been developed by several authors. Since Boag (1949), there has been extensive research, and parametric mixture models for the cure model have a long history (Farewell, 1982, 1986; Ghitany, Maller and Zhou 1994; Peng, Dear and Denham, 1998). Of particular note, the logistic regression model for the cure rate was introduced by Farewell (1982). The parametric assumptions (Weibull, log-Normal, generalized $\mathrm{F}$ and so on) for the latent distribution of uncured individuals have an important implication in estimation of the cure rate unless other concomitant information is available.

\footnotetext{
${ }^{*}$ Department of Biomedical Statistics, Graduate School of Medicine, Osaka University, 2-2 Yamada-oka, Suita, Osaka 565-0871, Japan E-mail: sugimoto@medstat.med.osaka-u.ac.jp

†Department of Biomedical Statistics, Graduate School of Medicine, Osaka University, 2-2 Yamada-oka, Suita, Osaka 565-0871, Japan

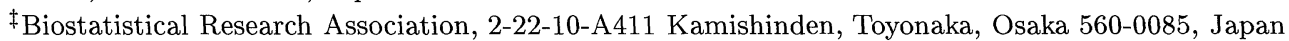

Key words: Cox's proportional hazards model; Cured individual; Logistic model; Martingale
} 
As for a regression model to explore the effects of covariates, it is not necessary to require a parametric distribution for the latency, since the regression variation on each covariate is of interest. Regarding such methods, recently nonparametric approaches for the latency have been proposed (Kuk and Chen, 1992; Taylor, 1995; Tsodikov, 1998; Sugimoto and Goto, 1999; Peng and Dear, 2000; Sy and Taylor, 2000). Kuk and Chen (1992), Sugimoto and Goto (1999), Peng and Dear (2000) and Sy and Taylor (2000) discuss an identical cure model, which is a semiparametric generalization of Farewell's model using Cox's proportional hazards model for the latency in the uncured group. Kuk and Chen (1992) proposed an estimation method involving Monte Carlo simulation. Sugimoto and Goto (1999), Peng and Dear (2000) and Sy and Taylor (2000) proposed an estimation method via the EM algorithm. In the former method, there is a loyalty as the semiparametric approach that a marginal partial likelihood can perfectly eliminate the nuisance baseline hazard function. However, the performance of parameter estimation by the Monte-Carlo method is not so much better when the sample size is larger, because the iterative number required in the exact computation is too enormous and feasible Monte-Calro approximations become rough. In the latter method, it is difficult to derive martingale properties because of some characteristics of the EM algorithm and the cure model, so that it has remained to establish a large sample theory and develop diagnosis tools based on martingale properties (see Lu and Ying (2004, p. 332)).

In this paper, we consider the same cure model as discussed by Kuk and Chen (1992), Sugimoto and Goto (1999), Peng and Dear (2000) and Sy and Taylor (2000), which we call "the PH cure model". We propose a new estimation method using a criterion named the pseudo partial likelihood. This method is enabled to give martingale approaches to check model assumptions and to establish a large sample theory. Also, we characterize the pseudo partial likelihood as a tool to estimate parameters, which provides the basis for developing diagnosis tools based on martingale residuals similar to the standard Cox model. We perform simulation studies to investigate how much the proposed method is appropriate for practical use, compared with other methods. One example from breast cancer data is given to illustrate the proposed method.

\section{The PH cure model}

In the PH cure model, information of the form $\left(T_{i}, \Delta_{i}, X_{i}, Z_{i}\right)$ for $i=1, \ldots, n$ is available. $T_{i}$ denotes the observable random variable $\min \left(T_{i}^{*}, U_{i}\right)$, where $T_{i}^{*}$ and $U_{i}$ are the random variables of true survival and censoring, respectively. $\Delta_{i}$ denotes the censoring indicator $I\left(T_{i}^{*} \leq U_{i}\right)$, where $I(\cdot)$ is the indicator function. $X_{i}$ and $Z_{i}$ are the covariate vectors related to cure rate and uncured survival, respectively. Also, as usual, $\left(T_{i}, \Delta_{i}\right)$ can be expressed as $\left(N_{i}(\cdot), Y_{i}(\cdot)\right)$ by the counting process and the at-risk process, where $N_{i}(t)=I\left(T_{i} \leq t, \Delta_{i}=1\right), Y_{i}(t)=I\left(T_{i} \geq t\right)$.

Let $\lambda_{i}(\cdot)$ be the hazard function of the $i$-th individual. Suppose that $\lambda_{i}$ holds for the proportional hazards model if the $i$-th individual is uncured and $\lambda_{i}$ has zero-hazard otherwise. That is, we can write

$$
\lambda_{i}(t)=\lambda\left(t ; Z_{i}, G_{i}\right)=G_{i} \lambda_{0}(t) \exp \left(\beta^{\prime} Z_{i}\right), i=1, \ldots, n,
$$

where $G_{i}=I$ (the $i$-th individual is uncured), $\lambda_{0}(t)$ is the baseline hazard function and $\beta$ is the $p$-dimensional parameter vector corresponding to $Z_{i}$. Also let $c_{i}$ be the cure rate function of the $i$-th individual, $c_{i}=\operatorname{Pr}\left(G_{i}=0 \mid X_{i}\right)$. Suppose $c_{i}$ is the logistic model

$$
c_{i}=c\left(\alpha ; X_{i}\right)=\exp \left(\alpha^{\prime} X_{i}\right) /\left\{1+\exp \left(\alpha^{\prime} X_{i}\right)\right\},
$$


where $\alpha$ is the $q$-dimensional parameter vector corresponding to $X_{i}$. The usual form of $X_{i}$ is $\left(1, X_{i 1}, \ldots, X_{i q-1}\right)^{\prime}$ as $X_{i 0}=1$.

Usually, we cannot observe $G_{i}=0$ of being cured and can only know $G_{i}=1$ of being uncured when $\Delta_{i}=1$. For this reason, a marginal type of the full likelihood (Boag, 1949) is applied to the inference for the cure model. Then the logarithm of the marginal full likelihood in the $\mathrm{PH}$ cure model is written as

$l_{m f}\left(\theta, \Lambda_{0}\right)=\sum_{i=1}^{n}\left[\Delta_{i} \log \left\{\left(1-c_{i}\right) \lambda_{0}\left(T_{i}\right) \mathrm{e}^{\beta^{\prime} Z_{i}} S_{i}\left(T_{i}\right)\right\}+\left(1-\Delta_{i}\right) \log \left\{c_{i}+\left(1-c_{i}\right) S_{i}\left(T_{i}\right)\right\}\right]$,

where $\Lambda_{0}(t)=\int_{0}^{t} \lambda_{0}(s) d s, S_{i}(t)=\exp \left\{-\mathrm{e}^{\beta^{\prime} Z_{i}} \Lambda_{0}(t)\right\}, \theta=\left(\alpha^{\prime}, \beta^{\prime}\right)^{\prime}$. If some parametric model is applied to $\Lambda_{0}$, we can obtain the joint estimate of $\left(\theta, \Lambda_{0}\right)$ by maximizing $l_{m f}$. There are two methods in which a nonparametric model is applied to $\Lambda_{0}$ : one estimation method is proposed by Kuk and Chen (1992) and the other is proposed by Sy and Taylor (2000) and Peng and Dear (2000). We review the latter shortly in the next paragraph.

The method constructs a profile estimate of $\Lambda_{0}(t)$ by applying the EM algorithm to $l_{m f}$ using the idea of Taylor (1995). The $m$-th E-step algorithm transforms or decomposes $l_{m f}$ into a form of the complete-data log likelihood,

$$
\begin{aligned}
l_{f}^{E M}\left(\alpha \mid w^{b(m)}\right) & =\sum_{i=1}^{n}\left[\left\{1-w_{i}^{b(m)}\left(T_{i}\right)\right\} \log c_{i}+w_{i}^{b(m)}\left(T_{i}\right) \log \left(1-c_{i}\right)\right] \\
l_{f}^{E M}\left(\beta, \Lambda_{0} \mid w^{b(m)}\right) & =\sum_{i=1}^{n}\left[\Delta_{i} \log \lambda_{0}\left(T_{i}\right)+\Delta_{i} \beta^{\prime} Z_{i}+w_{i}^{b(m)}\left(T_{i}\right) \log S_{i}\left(T_{i}\right)\right],
\end{aligned}
$$

for a given $w^{b(m)}=\left(w_{1}^{b(m)}\left(T_{1}\right), \ldots, w_{n}^{b(m)}\left(T_{n}\right)\right)$, where $w_{i}^{b(m)}\left(T_{i}\right)=w_{i}^{b}\left(T_{i} ; \theta^{(m)}, \Lambda_{0}^{(m)}\right)$,

$$
w_{i}^{b}\left(t ; \theta, \Lambda_{0}\right)=\left\{\begin{array}{ll}
\left(1-c_{i}\right) S_{i}(t) /\left\{c_{i}+\left(1-c_{i}\right) S_{i}(t)\right\}, & \text { if } \Delta_{i}=0 \\
1, & \text { if } \Delta_{i}=1
\end{array}, \quad i=1, \ldots, n,\right.
$$

and $w^{b(m)}$ is computed by current parameter values $\left(\theta^{(m)}, \Lambda_{0}^{(m)}\right)(m \geq 0)$.

In the $m$-th M-step algorithm, we find that a Breslow's estimate (Breslow, 1972) of $\Lambda_{0}$

$$
\hat{\Lambda}_{0}^{b}\left(t \mid \beta, w^{b(m)}\right)=\sum_{\left\{i: T_{i} \leq t\right\}}\left\{\Delta_{i} / \sum_{j=1}^{n} Y_{j}\left(T_{i}\right) \mathrm{e}^{\beta^{\prime} Z_{j}} w_{j}^{b(m)}\left(T_{j}\right)\right\}
$$

can be utilized for the maximization of $l_{f}^{E M}\left(\beta, \Lambda_{0} \mid w^{b(m)}\right)$. Substituting $\hat{\Lambda}_{0}^{b}\left(t \mid \beta, w^{b(m)}\right)$ into $\Lambda_{0}$ of $l_{f}^{E M}\left(\beta, \Lambda_{0} \mid w^{b(m)}\right)$ leads to a form of $\log$ partial likelihood of $\beta$

$$
l_{p}^{E M}\left(\beta \mid w^{b(m)}\right)=\sum_{i=1}^{n} \Delta_{i}\left[\beta^{\prime} Z_{i}-\log \left\{\sum_{j=1}^{n} Y_{j}\left(T_{i}\right) \mathrm{e}^{\beta^{\prime} Z_{j}} w_{j}^{b(m)}\left(T_{j}\right)\right\}\right] .
$$

That is, the M-step of $l_{f}^{E M}\left(\beta, \Lambda_{0} \mid w^{b(m)}\right)$ on $\left(\beta, \Lambda_{0}\right)$ is replaced by maximizing $l_{p}^{E M}\left(\beta \mid w^{b(m)}\right)$ on only $\beta$ in cases where $\Lambda_{0}$ is a nonparametric nuisance. Therefore, we perform the M-step of $l_{m f}$ in the semiparametric estimation via the EM algorithm by maximizing $l_{f}^{E M}\left(\alpha \mid w^{b(m)}\right)$ and $l_{p}^{E M}\left(\beta \mid w^{b(m)}\right)$ on $\alpha$ and $\beta$, respectively.

Let $\theta^{(m+1)}=\left(\alpha^{(m+1)}, \beta^{(m+1)}\right)$ be the current estimate obtained by the M-step for given $w^{b(m)}$, then the current estimate $\Lambda_{0}^{(m+1)}$ of $\Lambda_{0}$ is written as $\hat{\Lambda}_{0}^{b}\left(\cdot \mid \beta^{(m+1)}, w^{b(m)}\right)$. For the next $(m+1)$-th E-step, $w^{b(m)}$ is updated to $w^{b(m+1)}$ by substituting $\left(\theta^{(m+1)}, \Lambda_{0}^{(m+1)}\right)$ into $\left(\theta, \Lambda_{0}\right)$ of $(1)$. Finally, $\left(\theta^{(\hat{m})}, \Lambda_{0}^{(\hat{m})}\right)$ which provides $\hat{m}=\operatorname{argmax}_{m=0,1, \ldots} l_{m f}\left(\theta^{(m)}, \Lambda_{0}^{(m)}\right)$ becomes an estimate of $\left(\theta, \Lambda_{0}\right)$ in a series of the EM-iteration.

Some problems included in this semiparametric estimation come about when we consider how to set an initial value $\left(\theta^{(0)}, \Lambda_{0}^{(0)}\right)$. Though Dempster, Laird and Rubin (1977) 
discovered the background that the EM-iteration does not make a marginal full likelihood decrease, the iteration may fall to a local solution on a complicated likelihood surface. The EM-iteration in the $\mathrm{PH}$ cure model is apt to converge to a local maximum solution as the model fit becomes more complicated. For this reason, we often need a method to confirm whether an estimate obtained by the EM-iteration indeed achieves the global maximum. Then, we may consider the direct search method such as a grid search, which, although the most primitive, is effective. The direct search is also useful when we search a suitable $\left(\theta^{(0)}, \Lambda_{0}^{(0)}\right)$ such that a Newton-Raphson procedure with the EM-iteration will not easily fail to converge. However, using various values of $\left(\theta^{(0)}, \Lambda_{0}^{(0)}\right)$ requires many high-dimensional functions corresponding to $\Lambda_{0}^{(0)}$, so that we cannot even easily execute a direct search.

When we pursue the problem further, we find that, from analogy of $\Lambda_{0}^{(m+1)}, \Lambda_{0}^{(0)}$ may be $\hat{\Lambda}_{0}^{b}\left(\cdot \mid \beta^{(0)}, w^{b(-1)}\right)$ but a definition of $w^{b(-1)}$ is not provided. One remedy is solving the equation $\Lambda_{0}^{(0)}=\hat{\Lambda}_{0}^{b}\left(\cdot \mid \beta^{(0)}, w^{b}\left(\cdot ; \theta^{(0)}, \Lambda_{0}^{(0)}\right)\right)$ on $\Lambda_{0}^{(0)}$ for given $\theta^{(0)}$. The equation is obtained by letting the left side of (2) be $\Lambda_{0}^{(0)}$ and giving $\left(\theta^{(0)}, \Lambda_{0}^{(0)}\right)$ in the right side. However, it will be difficult to obtain this solution in mathematical analysis and computational aspects since it potentially includes high-dimensional joint equations. Such difficulties provided one motivation for producing this paper.

\section{Pseudo partial likelihood}

We propose an alternative method to the semiparametric estimation based on the EM algorithm reviewed in the previous section. Both the methods are useful as mutual supplements.

\subsection{Derivation}

Suppose $\mathscr{F}_{t-}$ is the history just prior to $t$ except the information on $G_{i}, i=1, \ldots, n$. This history is a filtration formally written as

$$
\mathscr{F}_{t}=\sigma\left(N_{i}(s), N_{i}^{U}(s), X_{i}, Z_{i}: 0 \leq s \leq t, i=1, \ldots, n\right),
$$

where $N_{i}^{U}(t)=I\left(T_{i} \leq t, \Delta_{i}=0\right)$ is the censoring counting process and $\sigma(A)$ is the smallest $\sigma$-algebra generated by $A$. Then, we can obtain the following results on the conditional expectation of $d N_{i}(t)$ given $\mathscr{F}_{t-}$ under some usual conditions about censoring and covarites.

Proposition 1 If $T_{i}^{*}$ is independent of $U_{i}$ and the censoring density $\operatorname{Pr}\left(U_{i} \mid Z_{i}, G_{i}\right)$ and the conditional density $\operatorname{Pr}\left(Z_{i} \mid X_{i}, G_{i}\right)$ of covariates do not depend on the values of $G_{i}$, then we have

$$
\operatorname{Pr}\left(G_{i}=1 \mid \mathscr{F}_{t-}\right)= \begin{cases}w_{i}(t), & \text { if } Y_{i}(t)=1 \\ 1, & \text { if } Y_{i}(t)=0 \text { and } \Delta_{i}=1 \\ 1-c_{i}, & \text { if } Y_{i}(t)=0 \text { and } \Delta_{i}=0\end{cases}
$$

where

$$
w_{i}(t)=w_{i}\left(t ; \theta, \Lambda_{0}\right)=\left(1-c_{i}\right) S_{i}(t) /\left\{c_{i}+\left(1-c_{i}\right) S_{i}(t)\right\}, i=1, \ldots, n .
$$

Proposition 2 If $T_{i}^{*}$ is independent of $U_{i}$, then we have

$$
\mathrm{E}\left[d N_{i}(t) \mid G_{i}=1, \mathscr{F}_{t-}\right]=Y_{i}(t) \exp \left(\beta^{\prime} Z_{i}\right) d \Lambda_{0}(t), i=1, \ldots, n .
$$

Furthermore, if (3) and (4) hold, then we have

$$
\mathrm{E}\left[d N_{i}(t) \mid \mathscr{F}_{t-}\right]=w_{i}(t) Y_{i}(t) \exp \left(\beta^{\prime} Z_{i}\right) d \Lambda_{0}(t), i=1, \ldots, n .
$$


Especially, in every situation, we can show

$$
\mathrm{E}\left[d N_{i}(t) \mid \mathscr{F}_{t-}\right]=\operatorname{Pr}\left(G_{i}=1 \mid \mathscr{F}_{t-}\right) \mathrm{E}\left[d N_{i}(t) \mid G_{i}=1, \mathscr{F}_{t-}\right],
$$

so that the last result of Proposition 2 is obtained using (3) and (4).

In the filtration model $\mathscr{F}_{t}$, as a substitute for the direct use of the binary random variable $G_{i}$, we treat the expected variable of $G_{i}$ averaged on $\mathscr{F}_{t-}$. Regardless of $\Delta_{i}=1$ or 0 , we find the counting processes in the $\mathrm{PH}$ cure model have the intensity processes given by

$$
\Lambda_{i}^{a}(t)=\int_{0}^{t} w_{i}(s) Y_{i}(s) \exp \left(\beta^{\prime} Z_{i}\right) d \Lambda_{0}(s), i=1, \ldots, n .
$$

Also, when $\left(\theta, \Lambda_{0}\right)$ is known, using Proposition 2 and Fleming and Harrington (1991, Section 1.3) under the above usual conditions about censoring and covariates, we conclude the processes given by $N_{i}(t)-\Lambda_{i}^{a}(t), i=1, \ldots, n$ are $\mathscr{F}_{t}$-martingales.

The $\log$ (pseudo) full likelihood of counting processes constructed on the filtration model $\mathscr{F}_{t}$ is written as

$$
l_{p f}\left(\theta, \Lambda_{0}\right)=\sum_{i=1}^{n} \log \left[\left\{\lambda_{0}\left(T_{i}\right) \mathrm{e}^{\beta^{\prime} Z_{i}} w_{i}\left(T_{i}\right)\right\}^{\Delta_{i}} \exp \left\{-\int_{0}^{T_{i}} \mathrm{e}^{\beta^{\prime} Z_{i}} w_{i}(t) d \Lambda_{0}(t)\right\}\right] .
$$

If $\left(w_{1}, \ldots, w_{n}\right)$ is fixed, we find that a Breslow's estimate (Breslow, 1972)

$$
\hat{\Lambda}_{0}(t \mid \theta)=\sum_{\left\{i: T_{i} \leq t\right\}}\left\{\Delta_{i} / \sum_{j=1}^{n} Y_{j}\left(T_{i}\right) \mathrm{e}^{\beta^{\prime} Z_{j}} w_{j}\left(T_{i} ; \theta, \hat{\Lambda}_{0}\right)\right\}
$$

obtained from $l_{p f}$ can be used as a substitute for $\Lambda_{0}$ to simplify $l_{p f}$. Note the slight differences between this $\hat{\Lambda}_{0}$ and $\hat{\Lambda}_{0}^{b}$ of (2). The $l_{p f}$ in which $\Lambda_{0}$ is eliminated partially by the profile estimate is modified to

$$
l_{p p}\left(\theta, \hat{\Lambda}_{0}\right)=\sum_{i=1}^{n} \Delta_{i} \log \left\{\mathrm{e}^{\beta^{\prime} Z_{i}} w_{i}\left(T_{i} ; \theta, \hat{\Lambda}_{0}\right) / \sum_{j=1}^{n} Y_{j}\left(T_{i}\right) \mathrm{e}^{\beta^{\prime} Z_{j}} w_{j}\left(T_{i} ; \theta, \hat{\Lambda}_{0}\right)\right\} .
$$

We call $l_{p p}$ the pseudo partial likelihood, where the term of "pseudo" is used to distinguish this partial likelihood from that derived in Kuk and Chen (1992).

In the definition of Cox (1975), as Kuk and Chen (1992, p. 534) indicated, the derivation of $l_{p p}$ is more direct. Let $S_{i}^{a}(t)$ be the probability element that an individual $i$ survives more than time $t$, which is written as $S_{i}^{a}(t)=c_{i}+\left(1-c_{i}\right) S_{i}(t)$ in the PH cure model. Then we can define a hazard corresponding to $S_{i}^{a}(t)$ as

$$
\bar{\lambda}_{i}^{a}(t)=\left\{-\partial S_{i}^{a}(t) / \partial t\right\} / S_{i}^{a}(t) .
$$

The conditional probability given by

$$
\bar{\lambda}_{i}^{a}\left(T_{i}\right) / \sum_{j=1}^{n} Y_{j}\left(T_{i}\right) \bar{\lambda}_{j}^{a}\left(T_{i}\right)
$$

is one element of an exact partial likelihood in Cox (1975) though the nuisance $\lambda_{0}(\cdot)$ cannot be eliminated completely. This probability forms the $i$-th element of $\exp \left\{l_{p p}\left(\theta, \Lambda_{0}\right)\right\}$. Specifically, $\exp \left\{l_{p p}\left(\theta, \Lambda_{0}\right)\right\}$ becomes the usual partial likelihood without $\lambda_{0}(\cdot)$ when $c_{i}=0$, $i=1, \ldots, n$. 


\subsection{Estimation of the hazard function}

The estimate of the (cumulative) baseline hazard function is obtained by solving the equation (5) on $\hat{\Lambda}_{0}$. Given $\hat{\Lambda}_{0}\left(t_{-}\right)$, the computation to get the next $\hat{\Lambda}_{0}(t)$ is achieved by finding $\hat{\lambda}_{0}(t)$ which satisfies $\left.F\right|_{\lambda_{0}(t)=\hat{\lambda}_{0}(t)}=0$, obtained from differentiating (5) with respect to time $t$, where $F$ is

$$
F\left(\lambda_{0}(t)\right)=\lambda_{0}(t) d(t)-d \bar{N}(t) / \sum_{j=1}^{n} Y_{j}(t) \mathrm{e}^{\beta^{\prime} Z_{j}} w_{j}\left(t ; \theta,\left\{\hat{\Lambda}_{0}\left(t_{-}\right)+\lambda_{0}(t) d(t)\right\}\right),
$$

$t_{-}$is a time prior to $t, \bar{N}(t)=\sum_{i=1}^{n} N_{i}(t)$ and $d(t)=t-t_{-}$. Let $\left(T_{(1)}, T_{(2)}, \ldots, T_{(k)}\right)$ be the order statistic for observed failure times of $T_{1}, T_{2}, \ldots, T_{n}$; then we compute each $\hat{\lambda}_{0}\left(T_{(i)}\right)$ by setting $t=T_{(i)}$ and $t_{-}=T_{(i-1)}(i=1, \ldots, k)$ as $T_{(0)}=0$. In each computation, a NewtonRaphson procedure is used. As $\lambda_{0}\left(T_{(i)}\right)$ becomes larger, the first term of $F$ is monotonically increasing and the second term of $F$ is monotonically decreasing, so that the $\lambda_{0}\left(T_{(i)}\right)$ which satisfies $F=0$ is usually determined uniquely.

In the above-mentioned estimation, since we need $k$ times Newton-Raphson procedures, sometimes we may think to reduce the load of such procedures. Then, instead of $\hat{\Lambda}_{0}(t \mid \theta)$, we may use a modified estimate

$$
\underline{\Lambda}_{0}(t \mid \theta)=\sum_{\left\{i: T_{i} \leq t\right\}}\left\{\Delta_{i} / \sum_{j=1}^{n} Y_{j}\left(T_{i}\right) \mathrm{e}^{\beta^{\prime} Z_{j}} w_{j}\left(T_{i-} ; \theta, \hat{\Lambda}_{0}\right)\right\},
$$

where $T_{i-}$ is an observed failure time just prior to $T_{i}$ such that $T_{i-}=T_{(l-1)}$ for $T_{i}=T_{(l)}$. Though $\underline{\Lambda}_{0}$ may have more estimation bias than $\hat{\Lambda}_{0}$ in a small sample, we do not have to perform Newton-Raphson procedures to obtain $\underline{\hat{\Lambda}}_{0}$ at all. The performance of these estimators is evaluated in the simulation study in Section 4.

\subsection{Estimation method}

We discuss here the estimation method for general and zero-tail constraint cases.

\section{General Case}

By maximizing the pseudo partial likelihood $l_{p p}\left(\theta, \hat{\Lambda}_{0}\right)$ on $\theta$, we obtain estimates of the regression parameters. Then $\hat{\Lambda}_{0}$ in $l_{p p}$ is jointly computed for any given $\theta$ via the manner in Section 3.2. When we want to reduce the computational load, $\underline{\Lambda}_{0}$ is substituted for $\hat{\Lambda}_{0}$.

We use a Newton-Raphson procedure for the algorithm to maximize $l_{p p}\left(\theta, \hat{\Lambda}_{0}\right)$, so that the first and second derivatives of $l_{p p}\left(\theta, \hat{\Lambda}_{0}\right)$ are required. We give the first derivatives in Appendix A.1. Note that the first and second derivatives include the terms of $\partial \hat{\Lambda}_{0} / \partial \theta$ and $\partial^{2} \hat{\Lambda}_{0} / \partial \theta^{2}$. We can concretely write out $\partial \hat{\Lambda}_{0} / \partial \theta$ and $\partial^{2} \hat{\Lambda}_{0} / \partial \theta^{2}$ because they can be explained by the Volterra integral equations. However, even if we set $\partial \hat{\Lambda}_{0} / \partial \theta=0$ and $\partial^{2} \hat{\Lambda}_{0} / \partial \theta^{2}=0$ in computation of the derivatives of $l_{p p}\left(\theta, \hat{\Lambda}_{0}\right)$, some differences in convergence of NewtonRaphson procedures were hardly seen in our numerical experience. Also in the case of poor initial values, a simultaneous Newton-Raphson procedure on $\theta$ may easily fail to converge.

\section{Zero-tail Constraint Case}

To obtain a good estimate for $\theta$, Taylor (1995) and Sy and Taylor (2000) proposed setting $S_{i}\left(T_{(k)+}\right)=0$ (referred to as the zero-tail constraint), where $T_{(k)+}$ is a time just after the last failure time. Peng (2003) also studied some modified versions of the zero-tail constraint in detail. For the same reasons given by them, it is also important to impose the zero-tail constraint in the pseudo partial likelihood method.

Note that $l_{p p}\left(\theta, \hat{\Lambda}_{0}\right)$ do not have any direct contribution from $S_{i}\left(T_{(k)+}\right)$. For this reason, we consider use of $l_{f}^{E M}\left(\alpha \mid w^{b}\right)$ within the framework of the pseudo partial likelihood. Then, 
we can obtain estimates of $\theta$ by maximizing $l_{p p}\left(\theta, \hat{\Lambda}_{0}\right)$ over $\theta$ under $\partial l_{f}^{E M}\left(\alpha \mid \hat{w}^{b}\right) / \partial \alpha=0$, where $\hat{w}^{b}=\left(\hat{w}_{1}^{b}\left(T_{1}\right), \ldots, \hat{w}_{n}^{b}\left(T_{n}\right)\right), \hat{S}_{i}(t)=\exp \left\{-\mathrm{e}^{\beta^{\prime} Z_{i}} \hat{\Lambda}_{0}(t)\right\}$ and each $\hat{w}_{i}^{b}(t)$ is written as

$$
\hat{w}_{i}^{b}(t)= \begin{cases}0, & \text { if } \Delta_{i}=0 \text { and } t>T_{(k)}, \\ \left(1-c_{i}\right) \hat{S}_{i}(t) /\left\{c_{i}+\left(1-c_{i}\right) \hat{S}_{i}(t)\right\}, & \text { if } \Delta_{i}=0 \text { and } t \leq T_{(k)}, \\ 1, & \text { if } \Delta_{i}=1 .\end{cases}
$$

We can approach the constrained optimization problem in various ways, for example by the Lagrange multiplier method or the projection method. Here, we provide a relatively stable algorithm based on the projection method in Appendix A.2.

\section{Numerical analysis}

\subsection{Simulation}

We performed simulation studies to evaluate the estimation method of the pseudo partial likelihood proposed in Section 3 (referred to as the PPL) compared with the method proposed by Sy and Taylor (2000) or Peng and Dear (2000) (referred to as the PRL-EM) and the method of the full parametric model (referred to as the Exp parametric).

Using a simulation design similar to Kuk and Chen (1992) or Peng and Dear (2000), we generated a dataset of a control group and a treatment group with $n / 2$ patients in each, where we consider cases of sample sizes $n=100$ and $n=300$. The indicator of the treatment group is the only covariate involved $\left(X_{i 1}=Z_{i}=0\right.$ or $\left.1, i=1, \ldots, n\right)$. We set $\left(\alpha_{0}, \alpha_{1}\right)=$ $(-0.5+0.5 a,-0.5+0.5 b)(a, b=0,1,2)$. These levels of $\left(\alpha_{0}, \alpha_{1}\right)=(-0.5,-0.5),(-0.5,0)$, $(-0.5,0.5),(0,-0.5),(0,0),(0,0.5),(0.5,-0.5),(0.5,0)$ and $(0.5,0.5)$ correspond to cure rate levels of $(38 \%, 27 \%),(38 \%, 38 \%),(38 \%, 50 \%),(50 \%, 38 \%),(50 \%, 50 \%),(50 \%, 62 \%)$, $(62 \%, 50 \%),(62 \%, 62 \%),(62 \%, 73 \%)$ in the control group versus the treatment group, respectively. The standard exponential distribution was used as the baseline distribution for uncured patients in the control group. We used $\beta=\log (1 / 2) \doteq-0.693$. The censoring times were generated according to an exponential distribution with mean time $1 / 0.28$. Thus, the expected censoring rates in cases of $\left(\alpha_{0}, \alpha_{1}, \beta\right)=(-0.5,-0.5,-0.693),(-0.5,0,-0.693)$, $(-0.5,0.5,-0.693),(0,-0.5,-0.693),(0,0,-0.693),(0,0.5,-0.693),(0.5,-0.5,-0.693),(0.5$, $0,-0.693)$ and $(0.5,0.5,-0.693)$ were $(51 \%, 53 \%),(51 \%, 60 \%),(51 \%, 68 \%),(61 \%, 60 \%)$, $(61 \%, 68 \%),(61 \%, 76 \%),(71 \%, 68 \%),(71 \%, 76 \%),(71 \%, 83 \%)$ for the control group versus the treatment group, respectively. The samples generated here sometimes included cases in which there were not any patients censored beyond the last failure time in either the control group or treatment group when $n$ is small. In such sample data, estimates of $\alpha_{1}$ tend to go to infinity in some directions because of binary covariates. Although some agreement is usually needed for an infinite estimate of $\alpha_{1}$, we neglect such sample data.

Under these settings, we generated the simulated data until 1000 samples were obtained. We used the zero-tail constraint in both the PPL and PRL-EM for the empirical reason why estimates obtained in a situation such that the covariates in $X$ and $Z$ are of binary type and have the same variations are apt to be unstable. In the Exp parametric, we did not use the zero-tail constraint because we assumed the right exponential model. As for starting values for the PRL-EM, we will be able to perform a realistic and suitable estimation by using $\left(\theta^{(0)}, \Lambda_{0}^{(0)}\right)$ corresponding to $w_{i}^{b(-1)}=\Delta_{i}(i=1, \ldots, n)$ (referred to as the PRL-EM (I)). To see this, we also use $\left(\theta^{(0)}, \Lambda_{0}^{(0)}\right)$ corresponding to $w_{i}^{b(-1)}=\Delta_{i}+\left(1-\Delta_{i}\right)\left(1-\left(l_{i}\right) / k\right)$, where $l_{i}$ is a maximal integer which satisfies $T_{\left(l_{i}\right)} \leq T_{i}\left(0 \leq l_{i} \leq k\right)$ (referred to as the PRL-EM (II)). In the PPL, we used $\underline{\hat{\Lambda}}_{0}$ instead of $\hat{\Lambda}_{0}$. We show the MSEs and biases of $\hat{\alpha}_{0}, \hat{\alpha}_{1}$ and $\hat{\beta}$ 
obtained from 1000 samples in Table 1 , where $\hat{\alpha}_{0}, \hat{\alpha}_{1}$ and $\hat{\beta}$ denote the estimates of $\alpha_{0}, \alpha_{1}$ and $\beta$.

As a whole, results from the three semiparametric methods do not look inferior relative to those from the Exp parametric which plays a role as control. This evidence gives us confidence in using these semiparametric methods. The PPL was superior to the PRL-EM in estimation of $\beta$, while the PRL-EM was superior to the PPL in estimation of $\alpha$. We verified this as well in the case of a larger sample size and other true parameters. However, these differences in the PPL and the PRL-EM are relatively small, so that the PPL is appropriate for practical use. Although the PRL-EM(I) and the PRL-EM(II) forced to start from initial values corresponding to the fixed $w^{b(-1)}$ are superior to the PPL in computational speed,

Table 1: MSE and bias of estimates of regression parameters from the PPL, PRL-EM (I), PRL-EM (II) and Exp Parametric

\begin{tabular}{|c|c|c|c|c|c|c|c|}
\hline \multirow{2}{*}{\multicolumn{2}{|c|}{$\begin{array}{r}\text { Sample Size } \\
\text { Method }\end{array}$}} & \multicolumn{3}{|c|}{$n=100$} & \multicolumn{3}{|c|}{$n=300$} \\
\hline & & $\hat{\alpha}_{0}$ & $\hat{\alpha}_{1}$ & $\hat{\beta}$ & $\hat{\alpha}_{0}$ & $\hat{\alpha}_{1}$ & $\hat{\beta}$ \\
\hline & & & True & $\left.\alpha_{0}, \alpha_{1}, \beta\right)$ & $-0.5,-0.5,-1$ & 693) & \\
\hline \multirow[t]{4}{*}{ MSE } & PPL & 0.1619 & 0.4611 & 0.0899 & 0.0505 & 0.1775 & 0.0383 \\
\hline & PRL-EM (I) & 0.1497 & 0.4325 & 0.1443 & 0.0486 & 0.1623 & 0.0524 \\
\hline & PRL-EM (II) & 0.1485 & 0.4321 & 0.1451 & 0.0482 & 0.1631 & 0.0514 \\
\hline & EXP Parametric & 0.1412 & 0.4911 & 0.1245 & 0.0469 & 0.1450 & 0.0463 \\
\hline \multirow[t]{5}{*}{ Bias } & PPL & -0.0561 & 0.0277 & -0.0101 & -0.0289 & -0.0469 & 0.0027 \\
\hline & PRL-EM (I) & -0.0363 & 0.1143 & 0.0416 & -0.0236 & 0.0390 & 0.0296 \\
\hline & PRL-EM (II) & -0.0438 & 0.1092 & 0.0420 & -0.0165 & 0.0250 & 0.0207 \\
\hline & EXP Parametric & -0.0122 & 0.1063 & 0.0495 & -0.0023 & 0.0486 & 0.0273 \\
\hline & & & True & $\left.\alpha_{0}, \alpha_{1}, \beta\right)$ & $-0.5,0,-$ & 693) & \\
\hline \multirow[t]{4}{*}{ MSE } & PPL & 0.1709 & 0.4216 & 0.1068 & 0.0497 & 0.1479 & 0.0413 \\
\hline & PRL-EM (I) & 0.1511 & 0.3784 & 0.1866 & 0.0461 & 0.1375 & 0.0578 \\
\hline & PRL-EM (II) & 0.1472 & 0.3735 & 0.1851 & 0.0456 & 0.1366 & 0.0576 \\
\hline & EXP Parametric & 0.1485 & 0.3562 & 0.1629 & 0.0454 & 0.1178 & 0.0528 \\
\hline \multirow[t]{5}{*}{ Bias } & PPL & -0.0495 & 0.0179 & 0.0298 & -0.0390 & -0.0305 & -0.0064 \\
\hline & PRL-EM (I) & -0.0089 & 0.0585 & 0.0368 & -0.0158 & 0.0224 & 0.0041 \\
\hline & PRL-EM (II) & -0.0177 & 0.0482 & 0.0328 & -0.0190 & 0.0208 & 0.0038 \\
\hline & EXP Parametric & 0.0021 & 0.0461 & 0.0402 & -0.0083 & 0.0048 & -0.0072 \\
\hline & & & True & $\left.0, \alpha_{1}, \beta\right)$ & $0.5,0.5,-$ & $.693)$ & \\
\hline \multirow[t]{4}{*}{ MSE } & PPL & 0.1637 & 0.3747 & 0.1464 & 0.0525 & 0.1224 & 0.0448 \\
\hline & PRL-EM (I) & 0.1467 & 0.3559 & 0.2266 & 0.0477 & 0.1226 & 0.0661 \\
\hline & PRL-EM (II) & 0.1418 & 0.3420 & 0.2240 & 0.0461 & 0.1170 & 0.0638 \\
\hline & EXP Parametric & 0.1429 & 0.3407 & 0.2099 & 0.0475 & 0.1201 & 0.0647 \\
\hline \multirow[t]{5}{*}{ Bias } & PPL & -0.0609 & 0.0577 & 0.1011 & -0.0430 & 0.0206 & 0.0307 \\
\hline & PRL-EM (I) & -0.0008 & 0.0589 & 0.0661 & -0.0149 & 0.0525 & 0.0443 \\
\hline & PRL-EM (II) & -0.0124 & 0.0387 & 0.0528 & -0.0184 & 0.0416 & 0.0370 \\
\hline & EXP Parametric & 0.0057 & 0.0126 & 0.0451 & -0.0107 & 0.0175 & 0.0205 \\
\hline & & & True & $\overline{\left.\chi_{0}, \alpha_{1}, \beta\right)}$ & $0,-0.5,-$ & 693) & \\
\hline \multirow[t]{4}{*}{ MSE } & PPL & 0.1445 & 0.4117 & 0.1442 & 0.0444 & 0.1408 & 0.0459 \\
\hline & PRL-EM (I) & 0.1346 & 0.3810 & 0.2327 & 0.0428 & 0.1313 & 0.0686 \\
\hline & PRL-EM (II) & 0.1361 & 0.3839 & 0.2345 & 0.0431 & 0.1299 & 0.0685 \\
\hline & EXP Parametric & 0.1333 & 0.3746 & 0.1985 & 0.0418 & 0.1164 & 0.0621 \\
\hline \multirow[t]{4}{*}{ Bias } & PPL & -0.0299 & -0.0129 & -0.0246 & -0.0282 & -0.0272 & 0.0129 \\
\hline & PRL-EM (I) & -0.0113 & 0.0688 & 0.0411 & -0.0109 & 0.0370 & 0.0292 \\
\hline & PRL-EM (II) & -0.0257 & 0.0554 & 0.0378 & -0.0165 & 0.0305 & 0.0275 \\
\hline & EXP Parametric & -0.0015 & 0.0423 & 0.0408 & -0.0029 & 0.0214 & 0.0183 \\
\hline
\end{tabular}


Table 1: (cont.)

\begin{tabular}{|c|c|c|c|c|c|c|c|}
\hline \multirow[b]{2}{*}{ MSE } & \multirow[b]{2}{*}{ PPL } & \multicolumn{6}{|c|}{ True $\left(\alpha_{0}, \alpha_{1}, \beta\right)=(0,0,-0.693)$} \\
\hline & & 0.1499 & 0.3844 & 0.1358 & 0.0459 & 0.1222 & 0.0481 \\
\hline & PRL-EM (I) & 0.1332 & 0.3505 & 0.2369 & 0.0435 & 0.1191 & 0.0719 \\
\hline & PRL-EM (II) & 0.1342 & 0.3469 & 0.2387 & 0.0442 & 0.1186 & 0.0719 \\
\hline & EXP Parametric & 0.1372 & 0.3410 & 0.2224 & 0.0435 & 0.1135 & 0.0690 \\
\hline \multirow[t]{5}{*}{ Bias } & PPL & -0.0539 & 0.0405 & 0.0325 & -0.0307 & -0.0145 & 0.0093 \\
\hline & PRL-EM (I) & -0.0147 & 0.0832 & 0.0505 & -0.0067 & 0.0294 & 0.0204 \\
\hline & PRL-EM (II) & -0.0338 & 0.0649 & 0.0414 & -0.0144 & 0.0158 & 0.0128 \\
\hline & EXP Parametric & -0.0105 & 0.0365 & 0.0327 & -0.0008 & -0.0016 & 0.0016 \\
\hline & & \multicolumn{6}{|c|}{ True $\left(\alpha_{0}, \alpha_{1}, \beta\right)=(0,0.5,-0.693)$} \\
\hline \multirow[t]{4}{*}{ MSE } & PPL & 0.1401 & 0.3622 & 0.1656 & 0.0451 & 0.1055 & 0.0555 \\
\hline & PRL-EM (I) & 0.1216 & 0.3453 & 0.2730 & 0.0405 & 0.1072 & 0.0891 \\
\hline & PRL-EM (II) & 0.1229 & 0.3347 & 0.2740 & 0.0414 & 0.1052 & 0.0885 \\
\hline & EXP Parametric & 0.1248 & 0.3432 & 0.2658 & 0.0405 & 0.1084 & 0.0872 \\
\hline \multirow[t]{5}{*}{ Bias } & PPL & -0.0725 & 0.1097 & 0.1140 & -0.0460 & 0.0120 & 0.0354 \\
\hline & PRL-EM (I) & -0.0197 & 0.1200 & 0.1057 & -0.0160 & 0.0382 & 0.0376 \\
\hline & PRL-EM (II) & -0.0459 & 0.0960 & 0.0862 & -0.0268 & 0.0166 & 0.0205 \\
\hline & EXP Parametric & -0.0186 & 0.0552 & 0.0671 & -0.0134 & 0.0019 & 0.0135 \\
\hline & & \multicolumn{6}{|c|}{ True $\left(\alpha_{0}, \alpha_{1}, \beta\right)=(0.5,-0.5,-0.693)$} \\
\hline \multirow[t]{4}{*}{ MSE } & PPL & 0.1436 & 0.3599 & 0.1194 & 0.0456 & 0.1260 & 0.0602 \\
\hline & PRL-EM (I) & 0.1369 & 0.3456 & 0.2578 & 0.0447 & 0.1236 & 0.0855 \\
\hline & PRL-EM (II) & 0.1411 & 0.3496 & 0.2623 & 0.0454 & 0.1240 & 0.0857 \\
\hline & EXP Parametric & 0.1354 & 0.3179 & 0.2389 & 0.0448 & 0.1203 & 0.0818 \\
\hline \multirow[t]{5}{*}{ Bias } & PPL & -0.0263 & -0.0117 & -0.0475 & -0.0130 & -0.0232 & 0.0170 \\
\hline & PRL-EM (I) & -0.0113 & 0.0737 & 0.0483 & 0.0060 & 0.0268 & 0.0286 \\
\hline & PRL-EM (II) & -0.0295 & 0.0499 & 0.0382 & -0.0008 & 0.0062 & 0.0176 \\
\hline & EXP Parametric & -0.0024 & 0.0246 & 0.0206 & 0.0123 & -0.0047 & 0.0093 \\
\hline & & \multicolumn{6}{|c|}{ True $\left(\alpha_{0}, \alpha_{1}, \beta\right)=(0.5,0,-0.693)$} \\
\hline \multirow[t]{4}{*}{ MSE } & PPL & 0.1470 & 0.3830 & 0.1686 & 0.0455 & 0.1119 & 0.0677 \\
\hline & PRL-EM (I) & 0.1350 & 0.3603 & 0.3539 & 0.0429 & 0.1127 & 0.1009 \\
\hline & PRL-EM (II) & 0.1390 & 0.3652 & 0.3632 & 0.0439 & 0.1135 & 0.1030 \\
\hline & EXP Parametric & 0.1383 & 0.3697 & 0.3302 & 0.0428 & 0.1102 & 0.0998 \\
\hline \multirow[t]{5}{*}{ Bias } & PPL & -0.0270 & 0.0192 & -0.0094 & -0.0265 & 0.0026 & 0.0233 \\
\hline & PRL-EM (I) & 0.0116 & 0.0621 & 0.0108 & -0.0016 & 0.0385 & 0.0279 \\
\hline & PRL-EM (II) & -0.0115 & 0.0325 & -0.0067 & -0.0110 & 0.0143 & 0.0108 \\
\hline & EXP Parametric & 0.0157 & -0.0038 & -0.0094 & 0.0048 & 0.0060 & 0.0010 \\
\hline & & \multicolumn{6}{|c|}{ True $\left(\alpha_{0}, \alpha_{1}, \beta\right)=(0.5,0.5,-0.693)$} \\
\hline \multirow[t]{4}{*}{ MSE } & PPL & 0.1487 & 0.4308 & 0.2336 & 0.0456 & 0.1242 & 0.0856 \\
\hline & PRL-EM (I) & 0.1333 & 0.4184 & 0.4124 & 0.0416 & 0.1248 & 0.1357 \\
\hline & PRL-EM (II) & 0.1395 & 0.4197 & 0.4299 & 0.0432 & 0.1266 & 0.1412 \\
\hline & EXP Parametric & 0.1374 & 0.4374 & 0.4113 & 0.0414 & 0.1289 & 0.1370 \\
\hline \multirow[t]{4}{*}{ Bias } & PPL & -0.0477 & 0.0857 & 0.1163 & -0.0300 & 0.0114 & 0.0283 \\
\hline & PRL-EM (I) & 0.0079 & 0.0957 & 0.0964 & 0.0035 & 0.0341 & 0.0202 \\
\hline & PRL-EM (II) & -0.0260 & 0.0596 & 0.0695 & -0.0102 & 0.0050 & -0.0048 \\
\hline & EXP Parametric & 0.0056 & 0.0205 & 0.0566 & 0.0090 & -0.0094 & -0.0201 \\
\hline
\end{tabular}

the PRL-EM(I) and the PRL-EM(II) do not often give the same results. Also, even if we used estimates obtained from the Exp parametric as starting values for the PRL-EM, we observed that the estimation performance did not necessarily improve. For these reasons, we should try many starting values for the PRL-EM, especially when we have complicated data or models. 


\subsection{Breast cancer data}

We consider the breast cancer data used first by Farewell (1986). The data consist of the time to relapse or death for three treatment arms of a clinical trial of adjuvant therapy for breast cancer involving 139 patients: 45 in controls, 51 in treatment A and 43 in treatment B. Farewell (1986) applied the Weibull cure model to investigate the effects of covariates on the cure rate and the uncured survival. Then Kuk and Chen (1992) and Peng and Dear (2000) studied this data set with the PH cure model. To estimate the parameters in the semiparametric model, the former used a Monte Carlo method, the latter used the PRL-EM with a zero-tail constraint. More recently, Peng (2003) presented the additional results from the PRL-EM with various zero-tail constraints.

Here, to investigate some differences between the PRL-EM and the PPL, we simply use the only information on three treatment arms as two binary covariates. As estimates of $\Lambda_{0}$, we consider Breslow's estimates, so that $\hat{\Lambda}_{0}^{b}$ in Section 2 for the PRL-EM and $\hat{\Lambda}_{0}$ or $\underline{\Lambda}_{0}$ in Section 3.2 for the PPL are used. And we apply the zero-tail constraints, which are suggested in Taylor (1995) for the PRL-EM and are described in Section 3.3.2 for the PPL. Under these settings, we estimate $\left(\theta, \Lambda_{0}\right)$ in the PRL-EM and the PPL and give its results in Table 2 and Figure 1, where we refer to the PPL in which $\hat{\Lambda}_{0}$ and $\underline{\hat{\Lambda}}_{0}$ are used as the PPL(a) and the PPL(b), respectively.

Results from the PPL(b) show relatively stronger similarity to those from the PRL-EM than to those from the PPL(a). Especially in estimation of $\beta$, the PPL(a) provides a larger negative effect of Treatment $\mathrm{A}$ and a smaller positive effect of Treatment $\mathrm{B}$ than those from the PRL-EM and the PPL(b), so that the estimated baseline hazard function from the PPL(a) has a larger value in the last failure time.

Also, to check how well these data were fitted in these methods, we sketch the estimated survival curves of three treatment groups from the PPL(a) and the PRL-EM with the Kaplan-Meier estimates in Figure 2. The estimated survival curves in Treatment A and Treatment B from the PPL(a) and the PRL-EM are seen to be almost overlapping on the interval 1900-2600 days, and the corresponding Kaplan-Meier estimates intersect at about 1900 days. The standard Cox model does not assume such crossing survival without timevarying covariates, while even if the Cox model with time-varying covariates is fitted, it will be often difficult to interpret the time-varying covariates. Then, for example if the time-varying effect is caused by cured events, the approach from the $\mathrm{PH}$ cure model will be useful as an alternative to the Cox model with time-varying covariates.

Table 2: Estimated parameters from the PPL and the PRL-EM for the breast cancer data

\begin{tabular}{|c|c|c|c|}
\hline \multicolumn{4}{|c|}{ Parameters for uncured survival } \\
\hline$\hat{\beta}$ & PRL-EM & PPL(a) & PPL(b) \\
\hline Treatment A & -0.313 & -0.808 & -0.490 \\
\hline Treatment B & 0.357 & 0.120 & 0.352 \\
\hline \multicolumn{4}{|c|}{ Parameters for cure rate } \\
\hline$\hat{\alpha}$ & PRL-EM & $\mathrm{PPL}(\mathrm{a})$ & $\mathrm{PPL}(\mathrm{b})$ \\
\hline Intercept & -0.089 & -0.043 & -0.101 \\
\hline Treatment A & 0.778 & 0.559 & 0.694 \\
\hline Treatment B & 1.034 & 0.960 & 1.040 \\
\hline
\end{tabular}




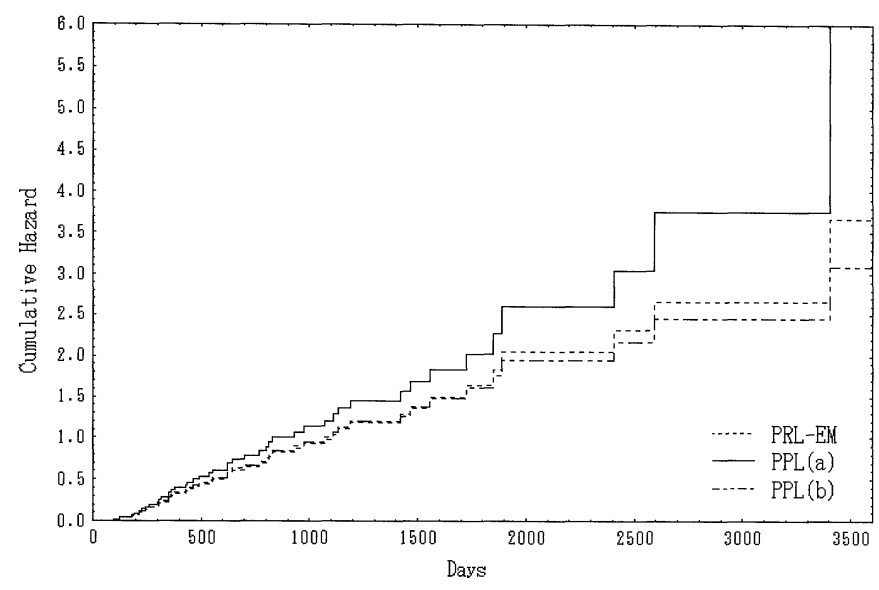

Fig. 1: Estimated cumulative baseline hazard functions from the PPL and the PRL-EM for the breast cancer data

\section{Discussion}

In this paper, we consider the same PH cure model as discussed by Kuk and Chen (1992), Sugimoto and Goto (1999), Peng and Dear (2000) and Sy and Taylor (2000) and propose a third estimation method derived from a character of the partial likelihood. Simulation studies show that the proposed method is reasonably appropriate for practical use. An application to breast cancer data is given to illustrate the proposed method.

Three semiparametric estimation methods for the $\mathrm{PH}$ cure model have some advantages and disadvantages relative to each other although there will not be essential differences in the estimation performances. Our method has a shorter computational time or does not depend on the number of Monte-Carlo samplings in comparison with Kuk and Chen's method. Also our method more easily computes the exact surface of the criterion function (the pseudo partial likelihood) in contrast with Peng and Dear's method and Sy and Taylor's method. This is because, to compute the exact surface of the criterion function (the observed likelihood) in the latter, we essentially need many values of the $(n-k)$-dimensional $w^{b(-1)}$. However, Kuk and Chen's method does not fail in convergence of a Newton-Rapson procedure so sensitively for some initial values of regression parameters as our method, and Peng and Dear's method and Sy and Taylor's method make a Newton-Rapson procedure converge with the shortest time (for one starting point $w^{b(-1)}$ ). In this sense, our method takes a middle position among the three methods. Therefore, elucidating further relationships between the three methods will lead to the more effective use of the PH cure model.

\section{Acknowledgements}

The authors thank Dr. Vern T. Farewell for providing the breast cancer data set in this research. Also, the authors are grateful to two referees and editors for their constructive comments and helpful suggestions. 

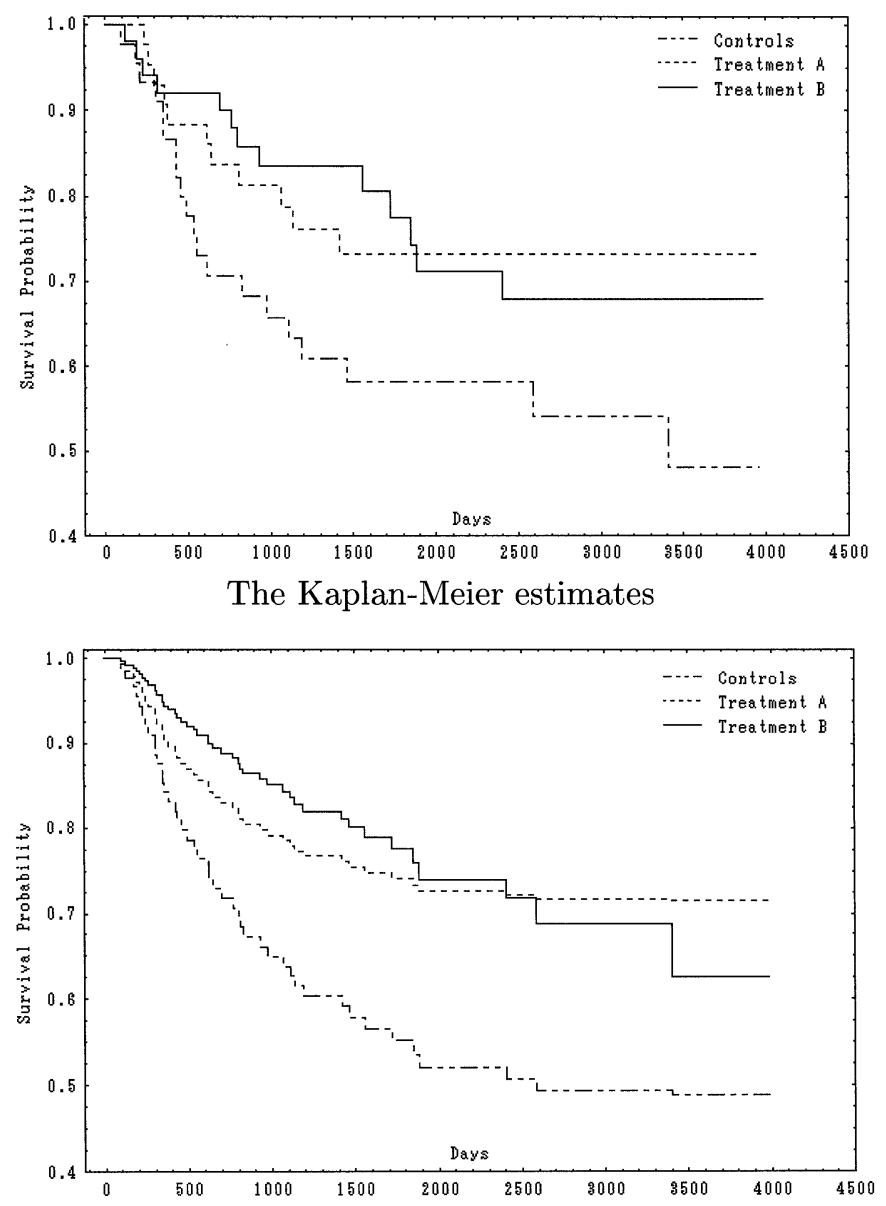

Estimated survival curves from the PPL(a)

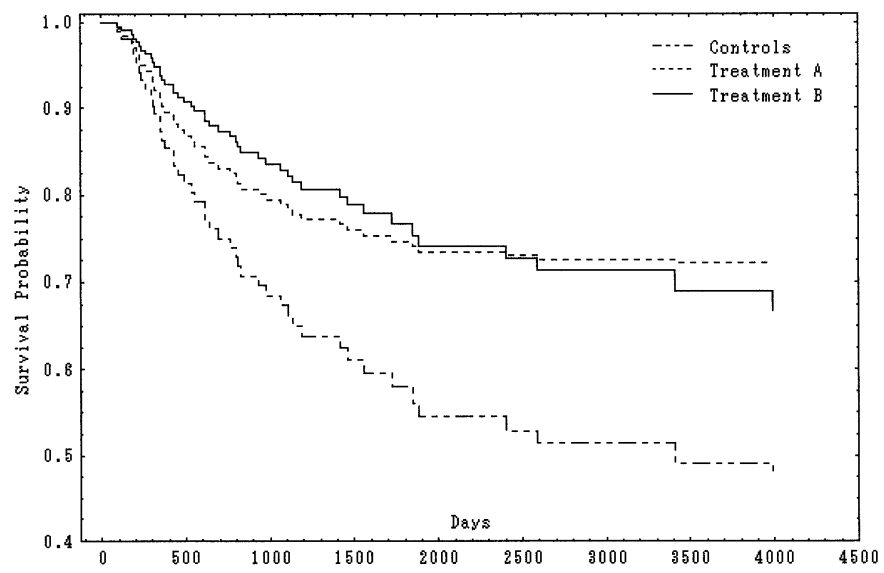

Estimated survival curves from the PRL-EM

Fig. 2: Estimated survival curves of three treatment groups for the breast cancer data 


\section{Appendix}

\section{A.1. The score functions}

Let $u$ be the vector of the score functions of $l_{p p}\left(\theta, \hat{\Lambda}_{0}\right)$, where $u=u\left(\theta, \hat{\Lambda}_{0}\right)=\left(u_{\alpha_{0}}, \ldots\right.$, $\left.u_{\alpha_{q}}, u_{\beta_{1}}, \ldots, u_{\beta_{p}}\right)^{\prime}$. The components of $u$ are

$$
\begin{aligned}
& u_{\alpha_{p}}\left(\theta, \hat{\Lambda}_{0}\right)=\sum_{i=1}^{n} \Delta_{i}\left[\tilde{X}_{i p}\left(T_{i}\right)-\frac{\sum_{j=1}^{n} Y_{j}\left(T_{i}\right) \tilde{X}_{j p}\left(T_{i}\right) \mathrm{e}^{\beta^{\prime} Z_{j}} \hat{w}_{j}\left(T_{i}\right)}{\sum_{j=1}^{n} Y_{j}\left(T_{i}\right) \mathrm{e}^{\beta^{\prime} Z_{j}} \hat{w}_{j}\left(T_{i}\right)}\right]-\sum_{i=1}^{n} \Delta_{i} \hat{\Lambda}_{\alpha_{p}}\left(T_{i}\right) \tilde{Q}_{i}\left(T_{i}\right), \\
& u_{\beta_{q}}\left(\theta, \hat{\Lambda}_{0}\right)=\sum_{i=1}^{n} \Delta_{i}\left[\tilde{Z}_{i q}\left(T_{i}\right)-\frac{\sum_{j=1}^{n} Y_{j}\left(T_{i}\right) \tilde{Z}_{j q}\left(T_{i}\right) \mathrm{e}^{\beta^{\prime} Z_{j}} \hat{w}_{j}\left(T_{i}\right)}{\sum_{j=1}^{n} Y_{j}\left(T_{i}\right) \mathrm{e}^{\beta^{\prime} Z_{j}} \hat{w}_{j}\left(T_{i}\right)}\right]-\sum_{i=1}^{n} \Delta_{i} \hat{\Lambda}_{\beta_{q}}\left(T_{i}\right) \tilde{Q}_{i}\left(T_{i}\right),
\end{aligned}
$$

where $\hat{w}_{i}(t)=\left.w_{i}(t)\right|_{\Lambda_{0}=\hat{\Lambda}_{0}}, \tilde{Z}_{i q}(t)=Z_{i q}-Z_{i q} \mathrm{e}^{\beta^{\prime} Z} \hat{\Lambda}_{0}(t)\left\{1-\hat{w}_{i}(t)\right\}, \tilde{X}_{i p}(t)=-X_{i p}\left\{1-\hat{w}_{i}(t)\right\}$,

$$
\tilde{Q}_{i}(t)=\mathrm{e}^{\beta^{\prime} Z_{i}}\left\{1-\hat{w}_{i}(t)\right\}-\frac{\sum_{j=1}^{n} Y_{j}(t) \mathrm{e}^{2 \beta^{\prime} Z_{j}}\left\{1-\hat{w}_{j}(t)\right\} \hat{w}_{j}(t)}{\sum_{j=1}^{n} Y_{j}(t) \mathrm{e}^{\beta^{\prime} Z_{j}} \hat{w}_{j}(t)}
$$

and $\hat{\Lambda}_{\theta_{l}}(t)=\partial \hat{\Lambda}_{0}(t) / \partial \theta_{l}$. Especially, we can calculate $\hat{\Lambda}_{\theta}(t)$ by solving some Volterra equations, which are obtained by differentiating (5). Also the second derivatives of $l_{p p}\left(\theta, \hat{\Lambda}_{0}\right)$ are obtained in the same manner.

\section{A.2. An algorithm for estimation under the zero-tail constraint}

We briefly outline an algorithm for the estimation method described in Section 3.3.2. Let $g(\theta)=\left(g_{1}, \ldots, g_{q}\right)^{\prime}$ and $g_{l+1}=\partial l_{f}^{E M}\left(\alpha \mid \hat{w}^{b}\right) / \partial \alpha_{l}$, then we have $g_{l+1}=\sum_{i} X_{i l}\left\{\hat{w}_{i}^{b}\left(T_{i}\right)-\right.$ $\left.\left(1-c_{i}\right)\right\}(l=0, \ldots, q-1)$. Let $H$ be the $q \times(q+p)$ matrix whose the $(i, j)$-th element is $\partial g_{i} / \partial \theta_{j}(i=1, \ldots, q ; j=1, \ldots,(q+p))$. Let $P=I-H^{\prime}\left\{H H^{\prime}\right\}^{-1} H$, then $P$ gives a projection matrix for the constraint $g(\theta)=0$, where $I$ is the identity matrix. And let $\underline{u}(\theta)=P u(\theta)$ for the vector $u$ of the score functions, then $\underline{u}(\theta)$ is used as a direction vector to maximize $l_{p p}\left(\theta, \hat{\Lambda}_{0}\right)$ jointly on $\theta$ under $g(\theta)=0$. However, the stability of convergence in this method is not up to par yet in our experience. So we develop a more stable method as follows. Let $\alpha(\beta)$ be a solution obtained by solving $g(\theta)=0$ on $\alpha$ for a fixed $\beta$, then the problem reduces to maximizing $l_{p p}\left(\beta, \alpha(\beta), \hat{\Lambda}_{0}\right)$ on only $\beta$. Hence, similarly to discussions on the profile likelihood inference, a direction vector $\tilde{u}_{\beta}$ to maximize $l_{p p}\left(\beta, \alpha(\beta), \hat{\Lambda}_{0}\right)$ on only $\beta$ under $g(\theta)=0$ can be written as $\tilde{u}_{\beta}=\underline{u}_{\beta}+\sum_{m} \underline{u}_{\alpha_{m}} \partial \alpha_{m} / \partial \beta$, where the term $\partial \alpha / \partial \beta$ is obtained by differentiating both sides of $g(\alpha(\beta), \beta)=0$ with respect to $\beta$.

Summarizing the above, we can use the following algorithm to maximize $l_{p p}\left(\theta, \hat{\Lambda}_{0}\right)$ under $g(\theta)=0$. Let $l=0$ and $\left(\epsilon_{1}, \epsilon_{2}\right)$ be a positive quantity.

1. Let $l=l+1$. Under given $\beta$ and $\hat{\Lambda}_{0}$, we find $\alpha^{(l)}(\beta)$ such that $\left.g(\alpha, \beta)\right|_{\alpha=\alpha^{(l)}(\beta)}=0$.

2. Under given $\beta$ and $\alpha^{(l)}(\beta)$, we update $\hat{\Lambda}_{0}$ by $(6)$. Go to 3 if $\left|\alpha^{(l)}(\beta)-\alpha^{(l-1)}(\beta)\right|<\epsilon_{1}$ and go to 1 otherwise.

3. Let $\beta^{a}=\beta$. Under given $\alpha^{(l)}(\beta)$ and $\hat{\Lambda}_{0}$, we update $\beta$ by using $\tilde{u}_{\beta}$, etc. Stop if $\left|\beta-\beta^{a}\right|<\epsilon_{2}$. Otherwise let $l=0$ and go to 1 . 


\section{REFERENCES}

Boag, J. W. (1949). Maximum likelihood estimations of the proportion of patients cured by cancer therapy. Journal of the Royal Statistical Society, Series B 11, 15-53.

Breslow, N. E. (1972). Contribution to discussion of paper by D. R. Cox. Journal of the Royal Statistical Society, Series B 34, 216-217.

Cox, D. R. (1972). Regression models and life tables (with discussion). Journal of the Royal Statistical Society, Series B 34, 187-220.

Cox, D. R. (1975). Partial likelihood. Biometrika 62, 269-276.

Dempster, A. P., Laird, N. M. and Rubin, D. B. (1977). Maximun likelihood from incomplete data via the EM algorithm (with discussion). Journal of the Royal Statistical Society, Series B 39, 1-38.

Farewell, V. T. (1982). The use of mixture models for the analysis of survival data with long-term survivors. Biometrics 38, 1041-1046.

Farewell, V. T. (1986). Mixture models in survival analysis: Are they worth the risk? Canadian Journal of Statistics 14, 257-262.

Fleming, T. R. and Harrington, D. P. (1991). Counting Processes and Survival Analysis. New York: John Wiley \& Sons.

Ghitany, M. E., Maller, R. A. and Zhou, S. (1994). Exponential mixture models with longterm survivors and covariates. Jounal of Multivariate Analysis 49, 218-241.

Kuk, A. Y. C. and Chen, C. H. (1992). A mixture model combining logistic regression with proportional hazards regression. Biometrika 79, 531-541.

Lu, W. and Ying, Z. (2004). On semiparametric transformation cure models. Biometrika 91, 331-343.

Peng, Y. (2003). Estimating baseline distribution in proportional hazards cure model. Computational Statistics and Data Analysis 42, 187-201.

Peng, Y. and Dear, K. B. G. (2000). A nonparametric mixture model for cure rate estimation. Biometrics 56, 237-243.

Peng, Y. and Dear, K. B. G. and Denham, J. W. (1998). A generalized $F$ mixture model for cure rate estimation. Statistics in Medicine 17, 813-830.

Sugimoto, T. and Goto, M. (1999). Adjustment of the proportional hazards model via characteristics of cases and its inference. Bulletin of the Computational Statistics of Japan 12, 85-106. (in Japanese)

Sy, J. P. and Taylor, J. M. G. (2000). Estimation in a cox proportional hazards cure model. Biometrics 56, 227-236.

Taylor, J. M. G. (1995). Semi-parametric Estimation in failure time mixture models. Biometrics 51, 899-907.

Tsodikov, A. (1998). A proportional hazard model taking account of long-term surviours. Biometrics 54, 1508-1516.

(Received July 2004, Accepted July 2005) 